Research Article

\title{
Game Chromatic Number of Generalized Petersen Graphs and Jahangir Graphs
}

\author{
Ramy Shaheen (D), Ziad Kanaya, and Khaled Alshehada \\ Department of Mathematics, Faculty of Science, Tishreen University, Lattakia, Syria \\ Correspondence should be addressed to Ramy Shaheen; shaheenramy2010@hotmail.com
}

Received 20 December 2019; Accepted 10 March 2020; Published 14 April 2020

Academic Editor: Kannan Krithivasan

Copyright (C) 2020 Ramy Shaheen et al. This is an open access article distributed under the Creative Commons Attribution License, which permits unrestricted use, distribution, and reproduction in any medium, provided the original work is properly cited.

Let $G=(V, E)$ be a graph, and two players Alice and Bob alternate turns coloring the vertices of the graph $G$ a proper coloring where no two adjacent vertices are signed with the same color. Alice's goal is to color the set of vertices using the minimum number of colors, which is called game chromatic number and is denoted by $\chi_{g}(G)$, while Bob's goal is to prevent Alice's goal. In this paper, we investigate the game chromatic number $\chi_{g}(G)$ of Generalized Petersen Graphs $G P(n, k)$ for $k \geq 3$ and arbitrary $n$, $n$-Crossed Prism Graph, and Jahangir Graph $J_{n, m}$.

\section{Introduction}

n 1981, Brams invented the game as a tool to provide a theoretical proof of the Four Color theorem without using computers [1]. But, it soon became apparent that there were many planar graphs which had game chromatic numbers greater than 4. In 1991, Bodlaender reinvented the game as a two-person game, Alice and Bob [2]. Players take turns coloring the vertices of a given finite graph $G=(V, E)$ and a color set $X=\{1,2, \ldots, k\}$. Alternately, players take turns over the elements of the set $V$; Alice starts the game, and her goal is to color the vertices with only $k=|X|$ colors, while Bob's goal is to prevent her. Bob wins the game if at any stage there are some vertices without available colors. The game chromatic number of a graph $G$, denoted by $\chi_{g}(G)$, is defined as the smallest integer $k$ such that Alice has a winning strategy on only $k$ colors. In [3], Faigle et al. proved that $\chi_{g}(T) \leq 4$ for a tree graph. In [4], Guan and Zhu introduced an upper bound to the game chromatic number of outerplanar graphs, which is $\chi_{g}(O P) \leq 7$. In [5], Bartnicki et al. studied the game chromatic number for the Cartesian product of two graphs. In [6], Sia studied the game for some families of Cartesian product graphs. Raspaud and $\mathrm{Wu}$ proved that $\chi_{g}\left(C_{2 m} C_{n}\right)=5 \longrightarrow \chi_{g}\left(C_{2 m} \square C_{n}\right)=5$ [7].
In [8], Shaheen et al. investigated the game chromatic number for some special circulant graphs and Generalized Petersen Graphs when $k=1,2,3$.

The Coloring Number was defined in [9] as the following:

Let $\Pi(G)$ be the set of linear orderings on the vertex set of a graph $G$, and let $L \in \Pi(G)$. For a vertex $v \in V(G)$, the back degree of $v$ with respect to $L$ is the number of its neighbors that precede it in $L$, i.e., $b(v) t n=q\left|h N_{L}^{+}(v)\right|$ $\longrightarrow b(v)=\left|N_{L}^{+}(v)\right|$ where $N_{L}^{+}(v)=\left\{u \in N(v)\right.$ and $\left.u<{ }_{L} v\right\}$. The back degree of $L$ is the maximum back degree of the vertices in $L$. The coloring number of $G$ denoted by $\operatorname{col}(G)$ is $1+b$ where $b$ is the minimum back degree of a linear ordering $L$ over all $L \in \Pi(G)$, i.e., $\operatorname{col}(G)=1+\min _{L \in \Pi(G)}$ $\max _{v \in \mathrm{V}(G)}(b(v))$. Clearly, $\chi(G) \leq \operatorname{col}(G)$.

The Game Coloring Number $\operatorname{col}_{g}(G)$ is the competitive version of the coloring number, which was introduced for the first time by $\mathrm{Zhu}$ in [10] to give an improved upper bound of the game chromatic number of planar graphs. The game coloring number is a two-person game, which is played by Alice and Bob, who alternate turns and with Alice starting first. After every move, a vertex is selected (among the remaining vertices) and is added to the end of the linear ordering which was formed by previous moves. After selecting all the vertices, a linear ordering $L$ of $V(G)$ is obtained, let $b$ be its back degree and then $\operatorname{col}_{g}(G)=1+b$. 
Alice's goal is to minimize the back degree $b$, while Bob's goal is to maximize it. Both players use their optimal strategies to form $L$.

A combinatorial game is a finite two-player game with perfect information and no chance. The game coloring number and the game chromatic number are combinatorial games [11].

In 2009, Kierstead and Kostochka were the first to present an application of coloring games to a nongame problem, which was the graph packing problem [12].

Several studies were presented in 2019 and discussed the game chromatic number of some specific graphs $[11,13,14]$. In [14], C. Chamberlin et al. investigated the game chromatic number of $G$ which is a graph produced by subdividing each edge of $G P(n, k)$ with $n$ large enough and $\operatorname{gcd}(n, k)=1$.

The uncolored vertex $v$ is called critical vertex if there is just one available color $c$ and there are some uncolored neighbors of $v$ which can be signed with $c$. In any stage of the game, if there are some critical vertices in Bob's turn, then he wins the game. Also, if there are critical vertices in Alice's turn, then she must defend them by her turn [7].

We denote the $i^{\text {th }}$ turn of Alice by $A_{i}$ and denote the process of assigning the vertex $v$ with the color $c$ by $v \longleftarrow c$.

Tow vertices are adjacent if there is an edge joining them together. For a vertex $v$ in the graph $G(V, E)$, the open neighbor set is defined as $N(v)=\{u \in V ; \exists u v \in E\}$ and the degree of $v$ is $\rho(v)=|N(v)| \longrightarrow \rho(v)=|N(v)|$. A graph $G=$ $(V, E)$ is $r$-regular if $\rho(v)=r$ for every $v \in V$.

Definition 1. Let the integer $k, n$ satisfy $k<n / 2$; Generalized Petersen Graph GP $(n, k)$ is the graph whose vertex set is $V \cup U$ where $V=\left\{v_{1}, \ldots, v_{n}\right\}$ and $U=\left\{u_{1}, \ldots, u_{n}\right\}$ and its edge set is $E=\left\{v_{i} v_{i+1}, v_{i} u_{i}, u_{i} u_{i+k}\right\}$, where $i=1,2, \ldots, n$ and subscripts are reduced modulo $n$.

Definition 2. For a positive even number $n \geq 4, n$-crossed prism graph $G=(V, E)$ is a graph obtained by taking two distinct cycles, outer cycle $C_{n}^{1}$ where $\mathrm{V}\left(C_{n}^{1}\right)=\left\{u_{1}, \ldots, u_{n}\right\}$, and the inner one $C_{n}^{2}$ where $\mathrm{V}\left(C_{n}^{2}\right)=\left\{v_{1}, \ldots, v_{n}\right\}$ and adding edges between them as $\left\{u_{i} v_{i+1}, u_{j} v_{j-1}\right\} ; i \in\{1,3, \ldots$, $n-1\}, j \in\{2,4, \ldots, n\}$.

Definition 3. Jahangir graph, $J_{n, m}$ for $m \geq 3$, is a cycle $C_{n m}$ with adding one vertex $v_{0}$ adjacent to $m$ vertices of $C_{n m}$ which are at distance $n$ from each other in $C_{n m}$.

Definition 4. The $n$-sunlet graph $S_{n}$ is a cycle $C_{n}$ where $\mathrm{V}\left(C_{n}\right)=\left\{u_{1}, u_{2}, \ldots, u_{n}\right\}$ with $n$ additional vertex $\left\{v_{1}, v_{2}, \ldots\right.$, $\left.v_{n}\right\}$, and $v_{i} u_{i} \in E\left(S_{n}\right)$ for $i=1, \ldots, n$.

Proposition 1 (see [10]). $\chi_{g}(G) \leq \operatorname{col}_{g}(G)$.

Proposition 2 (see [15]). The game chromatic number of the wheel graph $W_{n}$ is $\chi_{g}\left(W_{n}\right)=4$.

Theorem 1 (see [8]). For $n \geq 6$ and $k \in\{1,2,3\}$, we have $\chi_{g}(\operatorname{GPt}(n, k))=4 \longrightarrow \chi_{g}(G P(n, k))=4$.

\section{Main Results}

In this section, we investigate the game chromatic number of generalized Petersen graph, $n$-crossed prism graph, and Jahangir graph.

Lemma 1. For $n \geq 9$ and $k \geq 2$, every Generalized Petersen graph $G P(n, k)$ has $S_{8}$ as a subgraph like the graph in Figure 1.

The proof comes clearly from the definition of the Generalized Petersen graph.

Theorem 2. For $n \geq 9$ and $k \geq 4$, the game chromatic number of the Generalized Petersen Graph $G P(n, k)$ is $\chi_{g}(G P(n, k))=4$.

Proof. Generalized Petersen Graphs are 3-regular graphs; therefore, $\quad \chi_{g}(G P(n, k)) \leq \Delta+1=4$. For $k \in\{1,2,3\}$, $\chi_{g}(G P(n, k))=4$ is proven in Theorem 1 .

Now, by contradiction, we will prove that Player A does not have any winning strategy for any integer $k \geq 4$.

Suppose that we have a color set $C=\{1,2,3\}$; by using Lemma 2.1, we notice that, in $G P(n, k)$, there is the subgraph as the graph in Figure 1.

Whatever the vertex is chosen by player A to start with, we will get the same state, so without loss of generality assume that player $\mathrm{A}$ starts the game with $v_{1}$, i.e., $A_{1}: v_{1} \longleftarrow 1$ then $B_{1}: u_{k+1} \longleftarrow 2$; now, $u_{1}$ is a critical vertex so player A will defend it by coloring it with the third color or coloring its unique uncolored neighbor with a legal color, and whatever his choice for $A_{2}$ is, then $B_{2}: v_{k} \longleftarrow 1$ which means that the vertex $v_{k+1}$ will become a critical vertex and also player A will have to defend it so whatever his choice is for $A_{3}$ then $B_{3}: u_{n} \longleftarrow 2$ and now there are two critical vertices $\left\{u_{k}, v_{n}\right\}$ and player $\mathrm{A}$ cannot defend them together so three colors are not enough for the first player to have a winning strategy. Then, $3<\chi_{g}(G P(n, k))=4$.

Theorem 3. For $n \geq 6$, let $G$ be a $n$-crossed prism graph, and the game chromatic number of $G$ is $\chi_{g}(G)=4$.

Proof. $\chi_{g}(G) \leq 4$ since $\Delta(G)=3$; therefore, we are going to contradict that three colors are not sufficient. Let $X=\{1,2,3\}$ and $A_{1}: v_{1} \longleftarrow 1$; then, $B_{1}: v_{3} \longleftarrow 2$, and now, $v_{2}$ is a critical vertex so Alice is forced to defend it in only two ways. We will discuss Alice's second turn $A_{2}$ as follows:

Case 1. $A_{2}: v_{2} \longleftarrow 3$ then $B_{2}: u_{2} \longrightarrow u_{3}$, that gives two critical vertices $u_{2}, v_{4}$ and Alice cannot defend them at the same time

Case 2. $A_{2}: u_{1} \longleftarrow c$ where $c \in\{1,2\}$

Subcase 2.1. $c=1$ then $B_{2}: v_{n-1} \longleftarrow 2$ then $u_{n}, v_{n}$ will be critical vertices and Alice cannot defend them at the same time

Subcase 2.2. $c=2$ then $u_{2}$ will become a critical vertex during Bob's turn who will color $u_{3}$ with the color 3 , and Alice loses the game

That means $\chi_{g}(G)>3$, so $\chi_{g}(G)=4$. 


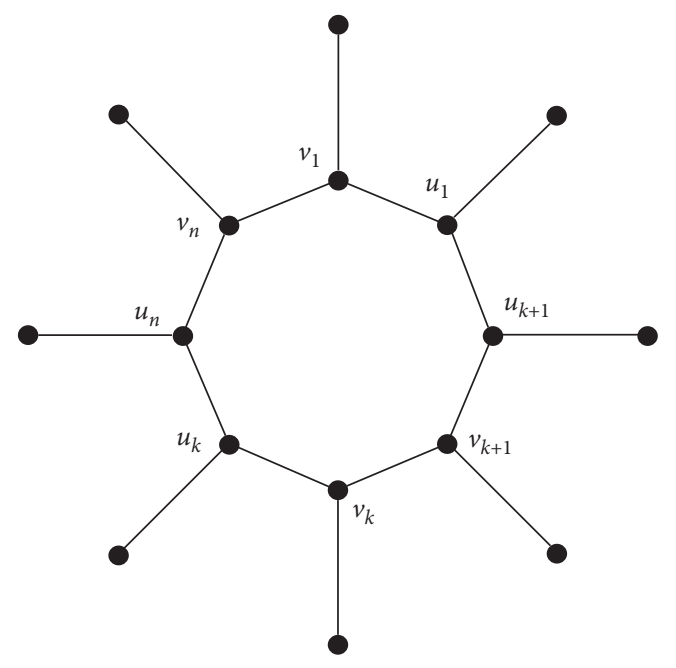

Figure 1: $S_{8}$ as subgraph of $G P(n, k)$.

Theorem 4. For $m \geq 3$, let $G \cong J_{n, m}$ be the Jahangir graph, and the game chromatic number

$$
\chi_{g}(G)= \begin{cases}4, & \text { if } n=1 \text { or }(n=2 \text { and } m \neq 3), \\ 3, & \text { if }(n=2 \text { and } m=3) \text { or }(n \geq 3 \text { and } m \geq 3) .\end{cases}
$$

Proof. First, because the Jahangir graph can be divided into cycles, then it is easy to show that $\chi_{g}\left(J_{n, m}\right)>2$. Now, we will discuss the following cases.

Case 1. $n=1$ or $(n=2$ and $m \neq 3)$

Subcase 1.1. $n=1$ then $G \cong W_{m}$, and using Proposition $1, \chi_{g}(G)=\chi_{g}\left(W_{m}\right)=4$.

Subcase 1.2. $n=2$ and $m \neq 3$ then three colors are not sufficient because if we suppose the color set $X=$ $\{1,2,3\}$ and $A_{1}: v_{0} \longleftarrow 1$ then $B_{1}: v_{2} \longleftarrow 2$, then there will be two critical vertices $v_{1}, v_{3}$, and Alice cannot defend them together on her next turn, so she loses the game.

Case 2. ( $n=2$ and $m=3$ ) or ( $n \geq 3$ and misarbitrary)

Subcase 2.1. When $n=2$ and $m=3$, as it is shown in Figure 2.

If $n=2$ and $m=3$, Alice can defend any critical vertices with three colors even with Bob's strategy which was mentioned in Subcase 1.2. We can explain Alice's winning strategy as follows: let the color set be $X=\{1,2,3\}$ and $A_{1}: v_{0} \longleftarrow 1$ then $B_{1}: v_{2} \longleftarrow 2$, so there will be two critical vertices $v_{1}, v_{3}$, and then when Alice chooses the next move to be $A_{2}: v_{5} \longleftarrow 3$; therefore, Bob cannot use the color 3 on the vertices $v_{4}, v_{6}$ to win the game and Alice wins with only three colors.

Subcase 2.2. When $n \geq 3$ and $m \geq 3$.

We will use the game coloring number as a tool to investigate the upper bound of $\chi_{g}\left(J_{n, m}\right)$.

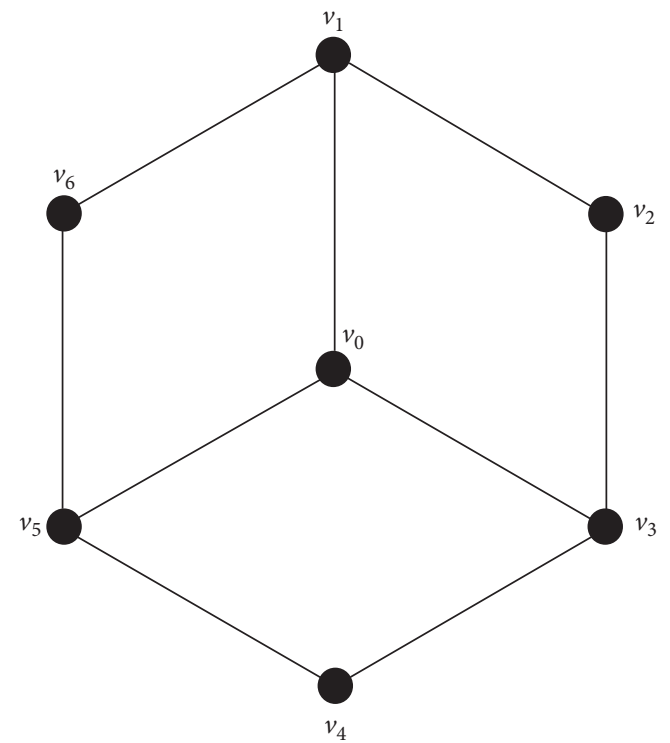

Figure 2: Jahangir graph $J_{2,3}$.

Let $D_{2}=\{v \in V \mid \rho(v)=2\}$ and $D_{3}=\{v \in V \mid \rho(v)=3\}$. The chosen vertex set is $C=\left\{v \in D_{3} \mid v\right.$ is a added to $\left.L\right\}$.

At the beginning, Alice chooses the central vertex $v_{0}$. Bob chooses a vertex $v \notin L$. Alice follows the next strategy to respond.

(1) If $v \in D_{2}$ and $\exists u \in N(v) \cap D_{3}$ and $u \notin L$ then Alice adds $u$ to $L$

(2) Else, if $v \in D_{2}$ and $\exists u \in D 3 C \longrightarrow \exists u \in D_{3} \backslash C$ then Alice chooses $u$ and adds it to $L$

(3) Else, Alice chooses any unchosen vertex $u$

Using this strategy on $J_{n, m}$ in case $n \geq 3$ produces an optimal linear order $L$ with back degree $b=$ $\max _{v \in L}\left|N_{L}^{+}(v)\right|=2$. So, $\operatorname{col}_{g}\left(J_{n, m}\right)=b+1=3$, and then, $\chi_{g}\left(J_{n, m}\right) \leq 3$ by using Proposition 1 . Therefore, $\chi_{g}\left(J_{n, m}\right)=3$ since $\chi_{g}\left(J_{n, m}\right)>2$.

\section{Conflicts of Interest}

The authors declare that they have no conflicts of interest.

\section{Acknowledgments}

This work was supported by Faculty of Science, Tishreen University, Syria.

\section{References}

[1] T. Bartnicki, J. Grytczuk, H. A. Kierstead, and X. Zhu, "The map-coloring game," The American Mathematical Monthly, vol. 114, no. 9, pp. 793-803, 2007.

[2] H. L. Bodlaender, "On the complexity of some coloring games," International Journal of Foundations of Computer Science, vol. 2, no. 2, pp. 133-147, 1991.

[3] U. Faigle, U. Kem, H. Kierstead, and W. T. Trotter, "On the game chromatic number of some classes of graphs," Ars Combinatorica, vol. 35, pp. 143-150, 1993. 
[4] D. J. Guan and X. Zhu, "Game chromatic number of outerplanar graphs," Journal of Graph Theory, vol. 30, no. 1, pp. 67-70, 1999.

[5] T. Bartnicki, B. Brešar, J. Grytczuk, M. Kovše, Z. Miechowicz, and I. Peterin, "Game chromatic number of Cartesian product graphs, Electron," Journal of Combinatorial Theory, vol. 15, 2008.

[6] C. Sia, "The game chromatic number of some families of Cartesian product graphs," AKCE International Journal of Graphs and Combinatorics, vol. 6, no. 2, pp. 315-327, 2009.

[7] A. Raspaud and J. Wu, "Game chromatic number of toroidal grids," Information Processing Letters, vol. 109, no. 21-22, pp. 1183-1186, 2009.

[8] R. Shaheen, Z. Kanaya, and K. Alshehada, "Game chromatic number of some regular graphs," Open Journal of Discrete Mathematics, vol. 9, no. 4, pp. 159-164, 2019.

[9] H. A. Kierstead and D. Yang, "Orderings on graphs and game coloring number," Kluwer Academic Publishers, vol. 10, pp. 255-264, 2003.

[10] X. Zhu, "The game coloring number of planar graphs," Journal of Combinatorial Theory, Series B, vol. 75, no. 2, pp. 245-258, 1999.

[11] A. Furtado, S. Dantas, C. de Figueiredo, and S. Gravier, "On caterpillars of game chromatic number 4," Electronic Notes in Theoretical Computer Science, vol. 346, pp. 461-472, 2019.

[12] H. A. Kierstead and A. V. Kostochka, "Efficient graph packing via game colouring," Combinatorics, Probability and Computing, vol. 18, pp. 765-774, 2009.

[13] M. S. Akhtar, U. Ali, G. Abbas, and M. Batool, "On the game chromatic number of splitting graphs of path and cycle," Theoretical Computer Science, vol. 795, pp. 50-56, 2019.

[14] C. Chamberlin, J. DeCapua, H. Elser, D. Gerraputa, and A. Hamm, "On game chromatic number analogues of Mycielsians and Brooks' Theorem," North Carolina Journal of Mathematics and Statistics, vol. 5, pp. 17-27, 2019.

[15] C. J. Destacamento, A. D. Rodriguez, and L. A. Ruivivar, The Game Chromatic Number of Some Classes of Graphs, DLSU Research Congress, De La Salle University, Manila, PH, USA, 2014. 